\title{
Probing Bow Shocks Around Exoplanets During Transits
}

\author{
A. A. Vidotto, M. Jardine, and C. Helling \\ SUPA, School of Physics and Astronomy, University of St Andrews, North Haugh, \\ St Andrews, KY16 9SS, UK \\ email: Aline.Vidotto@st-andrews.ac.uk
}

\begin{abstract}
Here, we summarise the conditions that might lead to the formation of a bow shock surrounding a planet's magnetosphere. Such shocks are formed as a result of the interaction of a planet with its host star wind. In the case of close-in planets, the shock develops ahead of the planetary orbit. If this shocked material is able to absorb stellar radiation, the shock signature can be revealed in (asymmetric) transit light curves. We propose that this is the case of the gas giant planet WASP-12b, whose near-UV transit observations have detected the presence of an extended material ahead of the planetary orbit. We show that shock detection through transit observations can be a useful tool to constrain planetary magnetic fields.
\end{abstract}

Keywords. planets and satellites: general - stars: winds, outflows - stars: coronae

The Earth hosts a bow shock that is formed around its magnetosphere as a result of its interaction with the solar wind. Analogously, from the interaction of an exoplanet with the coronal material of its host star, similar shock structures are expected to develop. A bow shock around a planet is formed when the relative motion between the planet and the stellar corona/wind is supersonic. The shock configuration depends on the direction of the flux of particles that arrives at the planet. If the dominant flux of particles impacting on the planet arises from the (radial) wind of its host star, the normal to the shock always points towards the star ("dayside-shock"). This is the case for a planet orbiting sufficiently distant from its host star, e.g., the Earth. If, on the other hand, the planet orbits very close to the star, the stellar wind velocity is much smaller than the Keplerian velocity $u_{K}$ of the planet. In this case, the dominant flux of particles impacting on the planet arises from the relative azimuthal velocity between the planetary orbital motion and the ambient plasma. The shock, therefore, forms ahead of the planetary orbit. The velocity of the particles that the planet 'sees' is supersonic if $\Delta u=\left|u_{K}-u_{\varphi}\right|>c_{s}$, where $u_{\varphi}$ is the azimuthal velocity of the stellar corona.

\section{WASP-12b's Magnetic Field}

WASP-12b orbits its host star (mass $M_{*}=1.35 M_{\odot}$, radius $R_{*}=1.57 R_{\odot}$ ) at an orbital radius of $a=3.15 R_{*}$ (Hebb et al. 2009) and the planet moves at a Keplerian orbital velocity of $u_{K}=\left(G M_{*} / a\right)^{1 / 2} \sim 230 \mathrm{~km} \mathrm{~s}^{-1}$ around the star. At such a close distance, the stellar wind should still present a low velocity. For simplicity, here we assume that this velocity is $\approx 0$ (more details can be found in Vidotto et al. 2010, 2011a, b). Therefore, stellar coronal material is compressed ahead of the planetary orbital motion, possibly forming a bow shock ahead of the planet. We believe this material is able to absorb enough stellar radiation, causing the early-ingress observed in the near-UV light curve (Fossati et al. 2010).

By measuring the phase difference between the beginnings of the near-UV and optical transits, Lai et al. (2010) derived the stand-off distance from the absorbing material 
(shock) to the centre of the planet: $r_{M} \simeq 4.2 R_{p}$. We take this distance to be the extent of the planetary magnetosphere. Pressure balance between the coronal total pressure and the planet total pressure requires that, at $r_{M}$,

$$
\rho_{c} \Delta u^{2}+\frac{\left[B_{c}(a)\right]^{2}}{8 \pi}+p_{c}=\frac{\left[B_{p}\left(r_{M}\right)\right]^{2}}{8 \pi}+p_{p}
$$

where $\rho_{c}, p_{c}$ and $B_{c}(a)$ are the local coronal mass density, thermal pressure, and magnetic field intensity, and $p_{p}$ and $B_{p}\left(r_{M}\right)$ are the planet thermal pressure and magnetic field intensity at $r_{M}$. Neglecting the kinetic term and the thermal pressures in Eq. (1.1), we have that $B_{c}(a) \simeq B_{p}\left(r_{M}\right)$. For dipolar stellar and planetary magnetic fields, we have

$$
B_{p}=B_{*}\left(\frac{R_{*} / a}{R_{p} / r_{M}}\right)^{3},
$$

where $B_{*}$ and $B_{p}$ are the magnetic field intensities at the stellar and planetary surfaces, respectively. Using the upper limit of $B_{*}<10 \mathrm{G}$ provided by Fossati et al. (2010b), our model predicts $B_{p}<24 \mathrm{G}$ for WASP-12b.

\section{Bow Shocks in Other Exoplanets?}

To extend the previous model to other transiting systems, near-UV data must be acquired. In Vidotto et al. (2011a), we presented a classification of the known transiting systems according to their potential for producing shocks that could cause observable light curve asymmetries. Once the conditions for shock formation are met, for it to be detected, it must compress the local plasma to a high enough density to cause an observable level of optical depth. Essentially, this classification is governed by the density $n$ of the medium surrounding the planet. Assuming a hydrostatic, isothermal corona

$$
\frac{n}{n_{0}}=\exp \left\{\frac{u_{K}^{2}}{c_{s}^{2}}\left[1-\frac{a}{R_{*}}\right]+\frac{v_{\text {rot }}^{2}}{c_{s}^{2}}\left[\frac{a}{R_{*}}-1\right]\right\},
$$

where $n_{0}$ is the density at the base of the corona and $v_{\text {rot }}$ is the stellar rotation velocity. We illustrate here the case where the coronal material is considered to be corotating with the star, such that $u_{\varphi}=v_{\text {rot }} a / R_{*}$. Therefore, the maximum temperature that still allow shock formation is such that $c_{s}=\left|u_{K}-v_{\text {rot }} a / R_{*}\right|$. With these assumptions, we estimate a minimum density required for shock formation through Eq. (2.1). We found that the most promising candidates to present shocks are: WASP-19b, WASP-4b, WASP-18b, CoRoT-7b, HAT-P-7b, CoRoT-1b, TrES-3, and WASP-5b.

We highlight that variations in the medium surrounding the plane, such as due to coronal mass ejections, the star's magnetic cycle, or even due to an eccentric planetary orbit, can cause temporal variations in the shock characteristics. Ultimately, this induces temporal variations in transit light curves (Vidotto et al. 2011b).

\section{References}

Fossati, L., Haswell, C. A., Froning, C. S., et al., 2010a, ApJ (Letters), 714, L222

Fossati, L., Bagnulo, S., Elmasli, A., et al., 2010b, ApJ, 720, 872

Hebb, L., Collier-Cameron, A., Loeillet, B., et al., 2009, ApJ, 693, 1920

Lai, D., Helling, Ch., \& van den Heuvel, E. P. J. 2010, ApJ, 721, 923

Vidotto, A. A., Jardine, M., \& Helling, Ch. 2010, ApJ (Letters), 722, L168

Vidotto, A. A., Jardine, M., \& Helling, Ch. 2011a, MNRAS (Letters), 411, L46

Vidotto, A. A., Jardine, M., \& Helling, Ch. 2011b, MNRAS, 414, 1573 